\title{
Patologia psiquiátrica nos Cuidados Primários de Saúde
}

João Amílcar Teixeira, ${ }^{*}$ Ana Sofia Cabral**

\section{RESUMO}

É consensual a alta prevalência de patologia psiquiátrica ao nível dos Cuidados de Saúde Primários. Consensual será também o papel fulcral que estes mesmos Cuidados desempenham enquanto primeiro filtro na abordagem dos problemas psiquiátricos, reconhecendo-se que, frequentemente, o Clínico Geral é o agente mais bem posicionado para uma correcta abordagem diagnóstica e terapêutica deste tipo de problemas. Assume-se, assim, como necessidade objectiva, que o Clínico Geral tome a seu cargo a maioria destes doentes e proceda à referenciação criteriosa duma minoria verdadeiramente necessitada de cuidados mais especializados. Em termos práticos, configura-se como prioritária uma progressiva melhoria da capacidade de actuação dos Clínicos Gerais, na área da Saúde Mental. É, portanto, neste contexto que surge a importância da articulação dos Serviços de Psiquiatria com os Centros de Saúde e a relevância do trabalho Consiliar e de Ligação do Psiquiatra junto dos Clínicos Gerais.

No presente artigo os autores tecem algumas considerações e lançam diversas questões sobre o tema em apreço, procurando abordar determinados aspectos centrais, nomeadamente, as particularidades de apresentação da patologia psiquiátrica nos Cuidados de Saúde Primários, em particular da «depressão», e decorrendo deste facto, as dificuldades na avaliação e abordagem das perturbações mentais neste setting particular.

Palavra-chave: Patologia Psiquiátrica; Cuidados de Saúde Primários; Psiquiatria Consiliar/Ligação.

\section{INTRODUÇÃO}

$\boldsymbol{Z}$ consensual a alta prevalência de patologia psiquiátrica ao nível dos Cuidados de Saúde Primários. Diversos estudos apontam neste sentido, desde Rosen e colaboradores ${ }^{1}$ (1972) e Regier, Goldberg e Taube ${ }^{2}$ (1978), até à actualidade. Consensual será também que a grande maioria é atendida nos Cuidados Primários e que apenas uma parte dos doentes, normalmente os mais graves, é assistida nos Serviços de Psiquiatria. O esquema de Goldberg e Huxley, ${ }^{3}$ tão divulgado na década de 80 , será bem elucidativo desta constatação. Segundo este modelo, da comunidade ao internamento hospitalar, o doente tem de atravessar diversos filtros passando por diferentes níveis de cuidados na área da Psiquiatria. O primeiro filtro é representado pela decisão de ir à consulta de Clínica Geral; o segundo filtro consiste na identificação ou reconhecimento pelo Clínico Geral da patologia psiquiátrica. Só depois aparece a decisão de encaminhar para a espe-

*Assistente Graduado de Psiquiatria, Serviço de Psiquiatria, Hospitais da Universidade de Coimbra.

**Interna do $5^{\circ}$ ano do Internato Complementar de Psiquiatria, Serviço de Psiquiatria, Hospitais da Universidade de Coimbra. cialidade, o que representa o terceiro filtro. Este último é tido como o menos permeável-apenas 1 em 20 doentes é encaminhado, isto é, cerca de $5 \%$ vai ao Psiquiatra, o que apenas reforça o já dito. Repare-se, assim, que a morbilidade psiquiátrica vista pelos respectivos especialistas, para além de «pequena», pode ser só uma atípica amostra do que se passa realmente nos Cuidados Primários e na comunidade. ${ }^{4}$

Bastariam estas razões de ordem epidemiológica para justificar a importância da dita patologia psiquiátrica nos Cuidados de Saúde Primários, e decorrendo desse facto, a importância da articulação dos Serviços de Psiquiatria com os Centros de Saúde e com os seus profissionais. Mas haverá ainda outra ordem de razões, nomeadamente, conceptuais e até organizativas. Por um lado, as primeiras, a que chamámos conceptuais, têm a ver com o modelo de doença, o que justifica também esta articulação. Para nós, e para muitos técnicos de Saúde Mental, apesar de não haver unanimidade, este modelo tem sempre no seu centro o doente, integrando a patologia apresentada na sua complexidade antropológica e vivencial, o que se sobrepõe ao plano meramente técnico do diagnóstico e do tratamento 
centrado nos sintomas e não na Pessoa. Logo, não se compadece de organicismos lineares. Nas razões de ordem organizacional realçamos apenas que a Organização Mundial de Saúde (OMS) advoga que o essencial para a melhoria dos Serviços de Psiquiatria e de Saúde Mental passa por um modelo de articulação e integração funcional de Cuidados Primários e Psiquiátricos. Isto sem esquecer uma clara diferenciação de um nível Primário e Secundário de Cuidados de Saúde em Psiquiatria, logo não perdendo a Psiquiatria o seu local próprio de actuação, como abordagem diferenciada e especializada. O papel do Clínico Geral/Médico de Família na linha da frente dos cuidados de saúde mental, com os técnicos de Psiquiatria funcionando como apoio e assistência fora do seu setting hospitalar habitual, é um aspecto chave para a OMS e para a Psiquiatria Consiliar e de Ligação (CL).

Para lançar algumas questões sobre este tema, vamos acrescentar ao que já foi dito certas particularidades e dificuldades na avaliação e abordagem das perturbações mentais nos Cuidados Primários e até na Psiquiatria em geral.

\section{DIFICULDADES NA ABORDAGEM DAS PERTURBAÇÕES MENTAIS}

Nos Cuidados Primários haverá obstáculos que radicam nas características deste contexto; nas características dos próprios médicos; e, finalmente, nas características da patologia encontrada, ou melhor, nas características dos doentes. No âmbito deste trabalho debruçar-nos-emos, em particular, sobre este último aspecto, mas sumariemos os outros dois, pois são indissociáveis.

\section{O contexto dos cuidados}

Refiram-se dificuldades que se prendem com: a) deficiências da formação pré e pós-graduada, prevalecendo ainda um modelo de etiologia meramente biológica de causalidade algo linear, o que será pouco adequado às pessoas doentes; b) o treino essencialmente em contexto hospitalar; c) a gama ampla de doentes, que não apenas os psiquiátricos, nos Cuidados Primários e o facto do Clínico Geral ter que lidar com perturbações emocionais dentro de cuidados médicos gerais; d) finalmente, a técnica diagnóstica, que habitualmente se concretiza em visitas breves e sucessivas, dirigindo-se primariamente ao alívio sintomático e assim adi- ando um diagnóstico integrativo dos dados biológicos, psicológicos e sociais.

\section{Os médicos}

Quanto às características dos médicos realcemos: a) a importância da personalidade de cada um, o que é apontado sempre como um dos factores mais relevantes; b) as atitudes negativas face à doença psiquiátrica e à importância de factores psicossociais na etiologia da doença; c) o tempo de prática clínica; d) as aptidões de comunicação; e) as técnicas e estilo de entrevista, (empatia). No fundo, questões que podem dificultar, a identificação e o tratamento de doentes com perturbações emocionais e/ou psiquiátricas nos Cuidados Primários.

\section{Os doentes}

Finalmente, quanto às características dos doentes, vejamos algumas questões prévias que dizem respeito à própria Psiquiatria e não apenas à Clínica Geral. Podemos até perguntar se para os psiquiatras será sempre claro o que é um «caso psiquiátrico», antes de concluirmos por dificuldades diagnósticas dos Clínicos Gerais. De facto, reparemos que, na Psiquiatria, os limites entre o normal e o patológico não são bem delimitados em algumas situações, o diagnóstico psiquiátrico não terá a exactidão de outras especialidades médicas e não haverá um modelo hegemónico. Finalmente, as perturbações psiquiátricas manifestam-se por modificações, menos objectiváveis, mas mais visíveis na interacção com os outros, no comportamento, na subjectividade da pessoa, sendo até vividos pelo próprio, no caso de patologias mais graves, como não sendo doença (negação da doença). ${ }^{5}$

Mas quanto ao contexto específico dos Cuidados Primários, parece haver questões particulares relativamente aos problemas de Saúde Mental.

Problemas Psicossociais - Antes de mais, repare-se que muitos destes problemas que poderão ser rotulados como psicológicos/emocionais/sociais ou, mais brevemente, como psicossociais, são muito mais frequentes que os «poucos» diagnósticos psiquiátricos «clássicos». Muitos doentes procuram ajuda por crises de vida, problemas de estilo de vida, envolvendo relações interpessoais, doenças físicas associadas a esses problemas e situações físicas que são influenciadas por factores psicossociais. Chega mesmo a avançar-se que 
pelo menos 33\% dos diagnósticos são de natureza psicossocial. ${ }^{6}$

Grassa ainda alguma confusão sobre estes problemas psicossociais. Não há certezas quanto à sua classificação ou nomenclatura e quanto ao seu curso, prognóstico e tratamento. Muitas destas situações não passarão de «reacções simples ao stress» (portanto, dentro do normal), ${ }^{7}$ significando apenas uma perturbação temporária pelas circunstâncias da vida. Apesar de serem abordados pelo Clínico Geral de uma forma diferente, tratá-los poderá ser patologizar o que não é patologia, não tendo lugar no DSM IVTR ${ }^{8}$ e até na ICD $10 .{ }^{9}$ Mesmo a perturbação de adaptação ou de ajustamento, que será a categoria diagnóstica que melhor poderia caracterizar muitas destas pessoas, não consegue abarcar completamente este vasto grupo. ${ }^{10}$

Sabemos também que há perturbação psicológica nos que reagem adversamente a acontecimentos ou crises de vida como o luto ou o divórcio, à doença física, nomeadamente a doença crónica ou até mesmo ao nascimento de um filho. Frequentemente, surgem sintomas que são reais, necessitando de apoio e aconselhamento ou de psicoterapia e/ou até de drogas psicotrópicas, o que não será muitas vezes abordado sem preconceitos. Goldberg, ${ }^{11}$ na tentativa de clarificar este vasto campo, afirma que na Clínica Geral encontramos fundamentalmente: 1 -Síndromes de perturbação psicológica que habitualmente não requerem intervenção porque normalmente são transitórios; 2 -Síndromes de perturbação psicológica/psiquiátrica que requerem intervenção, podendo esta ser a vários níveis: psicoterapia e/ou apoio social e/ou psicofarmacologia. Só nesta última circunstância é que estaríamos provavelmente a falar de situações psiquiátricas minor/perturbações de adaptação; 3 - Finalmente um último grupo constituído por situações psiquiátricas major onde é inequívoco o tratamento físico/farmacoterapia.

Na Clínica Geral, «faltam» ainda e frequentemente os sintomas para preencher todos os critérios clássicos das perturbações psiquiátricas, tratando-se muitas vezes de perturbações ligeiras, borderline ou sublimiares, segundo alguns em 13 a $30 \%$ dos doentes. Dito de outra forma, o cut off entre doença e não doença é frequentemente arbitrário. ${ }^{10}$

Isto leva alguns a questionar se não haverá necessidade de uma nova nosologia que contemple as «pato- logias» mais encontradas na Clínica Geral (embora não sabendo se estamos sempre perante patologia psiquiátrica). Isto fundamenta a preocupação sobre a validade do DSM e até da ICD para caracterizar a doença mental nos Cuidados Primários.

Realcemos, contudo, a importância e o avanço representado pelas actuais classificações tri-axiais e a defesa por alguns, como Costello, ${ }^{12}$ de uma investigação baseada nos sintomas e não nas síndromes, torneando, de alguma forma, a questão da classificação e permitindo avaliar melhor a diferença dimensional versus categorial entre situação psiquiátrica e normalidade. É crescente a convicção de que a doença mental não pode ser arrumada dentro de categorias definidas, apresentando-se antes a dimensionalidade como particularmente apropriada, sobretudo para as perturbações ditas neuróticas e da personalidade. ${ }^{9}$

Faltarão ainda critérios operacionais para designar elementos básicos da «evidência» observada na Clínica Geral que inclui sintomas relativamente pouco organizados, como a ansiedade, a depressão do humor, a tensão e a fadiga. Na ausência de critérios operacionais, ficamos sem uma ideia precisa sobre se são ou não diagnósticos. Nesta linha sabe-se, por exemplo, que $1 / 7$ da população sofre de sintomas ansiosos e depressivos. ${ }^{13}$

Estados Mistos de Ansiedade e Depressão - A ansiedade e a depressão serão as dimensões que melhor descrevem a maioria dos problemas mentais nos Cuidados de Saúde Primários, sendo habitualmente aceite que perturbações ansiosas/depressivas serão os diagnósticos mais frequentes. Isto leva alguns a falar de estados mistos de ansiedade e depressão na Clínica Geral com a ansiedade e a depressão coexistindo e sendo difíceis de separar. Existem sintomas físicos autonómicos em que os doentes se focam e estes quadros tenderão a cronificar como perturbações somatoformes se não forem tratados. Considera-se que os estados «puros» de doenças da ansiedade e afectivas são normalmente e apenas individualizadas pelo psiquiatra ${ }^{14}$ Refira-se que a DSM IV TR e a CID-10 contemplam a existência destes estados mistos ao nível da Clínica Geral, considerando-os como «intermédios», logo não preenchendo cabalmente os critérios quer de perturbação ansiosa quer afectiva, com as quais se deve fazer o diagnóstico diferencial. Mas, muitas vezes, ainda são diagnostica- 
dos como doenças da ansiedade, com prescrição apenas de ansiolíticos, o que não sustém o seu curso depressivo.

Estaremos provavelmente perante perturbações psiquiátricas a merecer melhor estudo e validação até se concluir pela sua existência enquanto «mera» doença da ansiedade ou afectiva, embora com apresentação ansiosa, ou antes pelo contrário enquanto grupo clínico distinto e autónomo, existindo dados que apontam nesse sentido. ${ }^{15,16}$

Depressão-Debrucemo-nos mais em pormenor sobre aquela que será a situação paradigmática do doente deprimido nos Centros de Saúde. Estima-se que esta realidade constitua cerca de $20 \%$ das consultas na Medicina Geral e Familiar e que a maioria vá primeiro ao médico de família, afirmando-se que apenas $1 / 3$ procura, inicialmente, o psiquiatra. Como já afirmado, há uma zona de sobreposição desta patologia com os chamados problemas psicossociais. ${ }^{17}$ Por outro lado, existe uma fronteira, difícil de delimitar, com a ansiedade sabendo que, para além dos já referidos estados mistos, é comum pelo menos esta co-morbilidade, o que pode contribuir para o obscurecimento da depressão em favor da ansiedade. Diz-se que $85 \%$ dos doentes com depressão experimentam sintomas significativos de ansiedade e que a comorbilidade de sintomas ou perturbações depressivas ocorre em mais de $90 \%$ dos doentes com perturbações de ansiedade. É também vulgar aceitar-se que esta coexistência de ansiedade em doentes deprimidos está associada com maior gravidade sintomatológica, curso crónico, maior comorbilidade médica/física e com pior recuperação. ${ }^{18}$

Alguns estudos afirmam que, na Clínica Geral, a depressão será menos grave, embora com uma vastíssima gama quanto à sua intensidade, será «menos psicologicamente centrada», mais determinada por factores ambienciais, mais relacionada com estilos de coping e sobretudo com uma maior apresentação somática. Aliás, a maioria dos autores diz-nos que os sintomas somáticos são a expressão mais comum de apresentação de toda a morbilidade psiquiátrica, nomeadamente da depressão.

Note-se que $65 \%$ dos doentes deprimidos se queixam de sintomas físicos com predomínio das queixas álgicas; ${ }^{19}$ mais de $2 / 3$ dos doentes deprimidos nos cuidados primários referem os sintomas somáticos como razão primária para a sua consulta ${ }^{20}$ («senha de admissão») mas em apenas $20 \%$ dos doentes deprimidos, que apresentam sintomas somáticos, é feito o diagnóstico. $^{21-22}$ Num outro estudo, $66 \%$ dos doentes não são diagnosticados como sofrendo de perturbação depressiva/ansiosa apesar de mais de 6 seis visitas por ano ao clínico geral por queixas somáticas. ${ }^{23}$

Verificamos, assim, que esta apresentação somática contribui para baixas taxas de reconhecimento da patologia depressiva focando-se o clínico geral quase exclusivamente nas queixas físicas, ${ }^{24-25}$ o que contribui para a designada «morbilidade psiquiátrica oculta».

Apesar deste bem estabelecido conhecimento psicopatológico, isto tem sido insuficientemente apreciado pelos sistemas diagnósticos oficiais, DSM IV TR e CID-10, apesar de reflectirem uma consciência crescente da importância destes sintomas somáticos na depressão ao incluir, entre os critérios diagnósticos, excessiva preocupação com saúde física e dores (cabeça, articulares, abdominais ou outras). Aliás, a questão do somático na depressão está incompletamente abrangida, pese embora o bem estabelecido fundamento somático do humor depressivo. Alguns autores afirmam que, quando queixas físicas dolorosas acompanham outros sintomas de depressão, o curso pode ser mais grave, com maior risco de recaída precoce, de cronicidade, de suicídio (ou até de mortalidade por causas naturais), de prejuízo no funcionamento e na qualidade de vida, e de aumento dos custos directa e indirectamente associados. ${ }^{25}$ Relativamente ao diagnóstico diferencial da depressão com sintomas somáticos predominantes, refiram-se as perturbações somatoformes, perturbações do espectro da ansiedade e até mesmo a própria esquizofrenia. Saliente-se, por fim, que o padrão de queixas somáticas é normalmente indistinguível entre doentes deprimidos e não deprimidos.

Doença Física e Perturbação Psiquiátrica - Se quisermos alargar a questão das relações entre doença física e perturbação psiquiátrica ou até da comorbilidade física/psiquiátrica, que aliás será a principal matéria do nosso trabalho em Psiquiatria $\mathrm{C} / \mathrm{L}$, referiríamos diversos tipos de interacções embora não estanques. Para além da perturbação psiquiátrica, como a depressão, que se apresenta com sintomatologia física e da depressão como reacção/adaptação a doença física, registem-se: perturbações psiquiátricas com complicações 
físicas (por exemplo, distúrbios do comportamento alimentar, alcoolismo,...), factores psicológicos que podem determinar morbilidade física (o campo tradicional da Psicossomática), a co-morbilidade de doença física e psiquiátrica meramente casual e, finalmente, as manifestações (neuro) psiquiátricas de doenças físicas (HIV, hipotiroidismo, ...). ${ }^{17}$ Alguns afirmam mesmo que a associação entre doença física e doença psiquiátrica nos cuidados primários e provavelmente noutros settings não será por acaso, chegando a falar-se aqui de uma propensão «psico-física» ao adoecer.

Isto não obsta a que os grandes quadros psiquiátricos clássicos se não encontrem nos Centros de Saúde, embora não possamos deixar de afirmar que também podem estar mascarados ou ocultados por aquilo que Barsky ${ }^{26}$ chama de «agendas escondidas».

Tratamento-Deixando o diagnóstico e passando ao tratamento, refira-se que a questão dos sintomas somáticos na depressão poderá ter implicações uma vez que os substratos neurobiológicos desses sintomas podem guiar outras abordagens terapêuticas. Contudo, repare-se que o simples «iniciar ab initium» de psicofármacos no tratamento da depressão não será consensual. E voltam-nos as perguntas: «de que falamos quando falamos de depressão» ou «o que são de facto os que chamámos de problemas psicossociais»? Com efeito, alguns autores advogam que uma espera atenta de duas semanas poderá evitar o tratamento porque poderão não ser «verdadeiras» depressões, embora outros discordem defendendo uma intervenção mais precoce de forma a evitar que o doente não volte à consulta ou deteriore o seu estado de saúde. Mas, de uma forma geral, as recomendações terapêuticas têm na sua base a gravidade do humor depressivo e os seus sintomas associados, embora não seja fácil a diferenciação, nomeadamente nos Cuidados Primários, entre depressão ligeira, moderada e grave. Consensual, no entanto, será que a referência para a Psiquiatria passará fundamentalmente pelo detectar de ideação de suicídio, história prévia relevante, sintomas psicóticos e bipolaridade.

Então como lidar com todos estes problemas, dos psicossociais aos psiquiátricos ${ }^{27} \mathrm{O}$ aconselhamento, a atitude terapêutica, que passa pela escuta empática, a promoção do apoio psicossocial (a começar pela família) e as psicoterapias formais serão de sublinhar. Refira-se, a título de exemplo, abordagens psicoterapêuti- cas alternativas como a sistémica, técnicas simples e breves orientadas para a resolução de problemas, psicoterapias breves modificadas, com eficácia comprovada na depressão nos Cuidados Primários e até genericamente as terapias cognitivas e comportamentais, não esquecendo o pragmatismo deste contexto de cuidados.

Mas o uso criterioso das drogas psicotrópicas será sempre de referir como crucial em muitas das situações, não esquecendo o avanço representado pelo aparecimento dos SSRI's no final da década de 80 e de outros anti-depressivos intervindo ao nível do sistema noradrenérgico e serotoninérgico, sem esquecer a importância neste setting das interacções medicamentosas, da polimedicação e do eventual abuso de ansiolíticos, hipnóticos, analgésicos e narcóticos. Repare-se que em Portugal, num estudo de Manuel Sousa ${ }^{28}$ parece haver uma prevalência maior de psicotrópicos prescritos relativamente a outros países, o que nos pode deixar a pensar no que isso significa.

Por fim, se fizermos um retrato-tipo do doente deprimido na Clínica Geral, apesar de este ser reducionista, diríamos: doente do sexo feminino, com um predomínio de queixas somáticas, sem queixas ansiosas e sobretudo depressivas aparentes ou evidentes, logo necessitando de ser sistematicamente pesquisadas, vivenciando acontecimentos de vida com significado stressor, com dificuldades de coping, com doença(s) física(s) associada(s) e que, apesar de ligeiras a moderadas, poderão cronificar se não forem tratadas precocemente e ou com psicotrópicos.

Saliente-se que, propositadamente, não foram abordados aspectos de Prevenção Primária porque mais do domínio da Saúde Pública. Também porque a Prevenção Primária em Saúde Mental será, em nossa opinião, questionável e limitada, devendo ser sempre humilde e cautelosa nos seus objectivos. O trabalho da Psiquiatria CLé fundamentalmente de Prevenção Secundária. Explicitando: é um esforço curativo, com identificação e abordagem precoces dos diversos factores implicados no adoecer, sem esquecer o stress representado pela própria doença e, obviamente, tendo que lidar com sintomas agudos já apontados, pesquisando-os com cuidado pois há sobreposições nem sempre claras. No fundo requer a observação do patológico, da personalidade e da situação. ${ }^{14}$ 


\section{CONCLUSÃO}

Em jeito de conclusão, e sem a preocupação de sermos exaustivos, mas antes a de continuarmos a lançar questões pertinentes para a nossa reflexão, muito do que foi referido alerta-nos para a importância da:

\section{Formação de psiquiatras e clínicos gerais}

Saliente-se a importância para o médico de um conhecimento detalhado da interacção entre doença psiquiátrica e doença física, podendo voltar a referir, a título de exemplo, os efeitos psicológicos/psiquiátricos da doença física, os aspectos psicológicos e físicos dos síndromes cerebrais orgânicos e a apresentação somática da doença psiquiátrica já amplamente referida. Isto não retira importância ao treino de aptidões de comunicação, por parte de todos os técnicos a trabalhar na área da Psiquiatria CL.

Nesta linha, pertinente seria: i. a inclusão no curriculum da formação médica do estudo da psicossomática, mais como atitude em Medicina do que como conjunto de patologias particulares; ii. a promoção da teoria e diversos estilos de prática CL como parte integrante do Internato de Psiquiatria; iii. o dotar dos Serviços de Psiquiatria com pessoal especialmente vocacionado para trabalho CL. No fundo, também em Psiquiatria CL a grande aposta e avanço tecnológico passa pela formação de profissionais competentes mas também motivados para as especificidades deste trabalho.

\section{Nosologia}

Partindo do que dissemos, há quem defenda a necessidade de uma nova nosologia que contemple não só as patologias psiquiátricas como também as «patologias» psicossociais. Repare-se que, tal como já referido, há uma progressiva sensibilidade a esta realidade por parte dos actuais sistemas classificativos internacionais.

Mas, será sempre importante que o próprio psiquiatra, no terreno, aprendendo e reflectindo com o médico de família sobre a sua própria prática, constate como os preceitos psiquiátricos podem ser alterados pela comorbilidade das disfunções médicas e psiquiátricas. Construir-se-á não uma nova Psiquiatria, enquanto teorização e constructos de referência, mas uma nova teoria da prática com uma nosologia algo própria que pode divergir da nosologia psiquiátrica clássica quando reconhecidamente se não adapta às novas realida- des da clínica nos Cuidados Primários.

\section{Modelos de prática psiquiátrica CL.}

Finalmente, no que diz respeito aos modelos e apesar da passagem dos anos, não será ainda consensual o que é a Psiquiatria CL e quais os modelos mais adequados para a sua prática. Ainda hoje nos EUA, a Psiquiatria de Ligação continua a ser associada ao trabalho psiquiátrico apenas em contexto de internamento hospitalar. Contudo, Gonzales e Norquist ${ }^{29}$ falam de Psiquiatria CL como qualquer intervenção clínica (C) ou educacional (L) para pessoal médico geral promovido por especialistas de Saúde Mental, vulgarmente, médicos, logo abrangendo os Cuidados de Saúde Primários. Na Liaison, e sem pormenorizar, poderíamos até incluir a importância do acautelar ou abordar alguma patologia psiquiátrica dos próprios técnicos envolvidos neste trabalho, o que passaria por exemplo pelo tão falado «burn out». Relativamente aos diferentes modelos, embora abertos a conclusões definitivas sobre a sua eficácia, parece que o psiquiatra na primeira linha dos Cuidados de Saúde Mental, substituindo os clínicos gerais, não constituirá a melhor solução, pelo menos, na relação custo-eficácia, para além do que o clínico geral deixaria de ser a pedra de toque desses cuidados na comunidade. Não haverá, portanto, em absoluto, dados que contradigam a justeza de um modelo em que o trabalho dos psiquiatras CL nos Centros de Saúde seja feito regularmente, ombro a ombro com o clínico geral, num estatuto de maior vulnerabilidade do que na prática psiquiátrica tradicional. Alguns doentes passariam a ser manejados apenas pelo clínico geral depois de abordados neste contexto CL. As referências para a psiquiatria seriam feitas após discussão ou aquele encontro face a face. Finalmente, quando fosse necessária esta referência para a psiquiatria, seria mandatário o posterior feed back para o médico de família.

Costuma-se afirmar que o mais importante para o sucesso das intervenções CL, para além dos seus diferentes modelos, será o estabelecer de uma relação de trabalho personalizada, «quase íntima», entre psiquiatra e médico de família.

O importante será aumentar a capacidade dos Serviços de Saúde, amplificando a capacidade de intervenção - do diagnóstico à terapêutica - e optimizando os cuidados à maioria das pessoas que adoecem mentalmente. 
O que se pretende serão efeitos directos destas intervenções CL e que se consubstanciariam num melhor e mais precoce reconhecimento e diagnóstico, numa mais adequada prescrição terapêutica, numa melhoria das referências para a especialidade e no encorajar de um papel mais activo, por parte dos médicos de família, no lidar com alguns doentes mentais. Mas, quanto aos efeitos indirectos, nomeadamente, a generalização das mudanças de comportamentos e atitudes dos clínicos gerais a todos os seus doentes, após terminada a intervenção CL, os diferentes estudos são pouco conclusivos. Mas repare-se que a avaliação compreensiva dos efeitos das intervenções CL não é limitada às mudanças de comportamento dos clínicos gerais, apesar de estas serem importantes. Há que ter também em linha de conta a qualidade do trabalho clínico, a relação custo eficácia e a aceitabilidade e satisfação por parte do doente.

As abordagens actuais sugerem que as mudanças duradouras podem requerer intervenções adicionais ou alternativas em direcção às pressões sentidas pelos clínicos gerais, como por exemplo, a falta de tempo ou aspectos relacionados com a aderência ao tratamento.

Em suma, e numa perspectiva optimista, se tudo passar pela avaliação sistemática, biológica, psicológica e social (uma vez que tecnologia e «compaixão» não são incompatíveis) ou, dito de outra forma, se tudo passar por cuidados compreensivos e multidisciplinares, muito teremos já conseguido. Com o atingir global dos objectivos da Psiquiatria CL, trabalharíamos para a «sua própria e feliz extinção». Entretanto, cuidar da pessoa doente na sua totalidade, contribuir para a formação contínua de todos os membros da equipa envolvida e fomentar a pesquisa e investigação clínica continuam a ser objectivos perseguidos..$^{30}$ As constantes da nossa atitude e da nossa prática nesta relação de trabalho da Psiquiatria CL serão: 1 . a procura da relação entre o soma e a psique; 2 . a actualização dos conhecimentos sobre patologização e terapêutica; 3. e sobretudo a doação de sentido humano à acção terapêutica. ${ }^{30}$

\section{REFERÊNCIAS BIBLIOGRÁFICAS}

1. Rosen $B$, Locke $B$, Goldberg $D$, Babigian $H$. Identification of emotional disturbance in patients seen in general medical clinics. Hospital and Community Psychiatry 1972 Dec; 23;129: 364-70.

2. Regier DA, Goldberg ID, Taube CA. The de facto U.S. mental health services system: a public health perspective. Arch Gen Psychiatry 1978
Jun; 35 (6): 685-93.

3. Goldberg D, Huxley P. Mental illness in the community: the pathway to psychiatric care. London: Tavistock Publications, 1980.

4. Goldberg D. The recognition of psychological illness by general practioners. In: Clare A, Lader M, editors. Psychiatry in General Practice. London: Academic Press; 1982. p. 35-41.

5. Jara J. Contribuição para um Livro Branco da Psiquiatria e da Saúde Mental em Portugal. Lisboa: Hospital Júlio de Matos; 2007.

6. Cassata D, Kirkman-Liff BL. Mental health activities of family physicians. J Fam Pract 1981 Apr; 12 (4): 683-92.

7. Pio Abreu JL, Teixeira JA. O caso posiquiátrico: limites e detecção. Psiquiatria Clínica 1984; 5 (2): 99-105.

8. American Psychiatric Association. Diagnostic and Statistical Manual of Mental Disorders. 4th ed rev. Washington DC:American Psychiatric Association; 2000.

9. World Psychiatric Association. International Classification and Diagnosis. 10th ed. Geneva:World Psychiatric Association; 1993.

10. Katerndahl DA, Larme AC, Palmer RF, Amodei N. Reflections on DSM classification and its utility in primary care: case studies in «mental disorders». Prim Care Companion J Clin Psychiatry 2005; 7(3): 91-9.

11. Goldberg D. The treatment of mental disorders in general medical settings. Gen Hosp Psychiatry 1992 Mar; 14 (2): 83-5.

12. Costello CG. Research on symptoms versus research on syndromes: arguments in favour of allocating more research time to the study of symptoms. Br J Psychiatry 1992 Mar; 160: 304-8.

13. Meltzer H, Gill B, Petticrew M. OPCS Surveys of Psychiatric Morbidity in Great Britain. Bulletin No. 1:The Prevalence of Psychiatric Morbidity among Adults Aged 16-64, Living in Private Households, in Great Britain. London: OPCS; 1994.

14. Lopes, G. Estados Mistos de Ansiedade e Depressão. In: Lopes G, editor. Temas CL de Psiquiatria, Psicossomática e Psicologia. Lisboa: Grupo Português de Psiquiatria Consiliar e de Ligação, 1999. p. 41-59.

15. Tarsia M, Power M, Sanavio E. Implicit and explicit memory biases in mixed anxiety-depression. J Affect Disord 2003 Dec; 77 (3): 213-25.

16. Montgomery S. Anxiety and Depression. Petersfield:Wrightson, 1993.

17. Carvalho S, Freitas JL. Depressão e Doença Médica. In: Lopes G, editor. Temas CL de Psiquiatria, Psicossomática e Psicologia. Lisboa: Grupo Português de Psiquiatria Consiliar e de Ligação; 1999. p. 69-91.

18. Gorman J. Comorbid depression and anxiety spectrum disorders. Depr Anxiety 1996-1997; 4 (4): 160-8.

19. Bair MJ, Robinson RL, Katon W, Kroenke K. Depression and pain comorbidity: a literature review. Arch Intern Med 2003 Nov 10; 163 (20): 2433-45.

20. Simon GE, Von Korff M, Piccinelli M, Fullerton C, Ormel J. An international study of the relation between somatic symptoms and depression. N Engl J Med 1999 Oct 28; 341 (8): 1329-35.

21. Kirmayer LJ, Robbins JM, Dworkind M, Yaffe MJ. Somatization and the recognition of depression and anxiety in primary care. Am J Psychiatry 1993 May; 150 (5): 734-41.

22. Kessler D, Lloyd K, Lewis G, Gray DP. Cross sectional study of symptom attribution and recognition of depression and anxiety in primary care. BMJ 1999 Feb 13; 318 (7181): 436-40.

23. Pearson SD, Katzelnick DJ, Simon GE, Manning WG, Helstad CP, Henk HJ. Depression among high utilizers of medical care. J Gen Intern Med 1999 Aug; 14 (8): 461-8. 
24. Robert $H$, Howland M, Michael E, Thase M. Comorbid depression and anxiety: when and how to treat. J Psychiatrt 2005 Aug; 329 (11): 891$-1047$.

25. Kapfhammer HP. Somatic symptoms in depression. Dialogues Clin Neurosci 2006; 8 (2): 227-39.

26. Barsky A. A research agenda for outpatient consultation-liaison psychiatry. Gen Hosp Psychiatry 1993 Nov; 15 (6): 381-5.

27. Teixeira J. Dificuldades na identificação do doente psiquiátrico nos cuidados primários de saúde. In: Lopes G, editor. Temas CL de Psiquiatria, Psicossomática e Psicologia. Lisboa: Grupo Português de Psiquiatria Consiliar e de Ligação, 1999. p. 27-41.

28. Sousa M. Perfil dos utilizadores de psicofármacos na Unidade de Saúde Familiar de Canelas. Rev Port Clin Geral 2007 Jan-Fev; 23 (1): 33-42.

29. Gonzalez J, Norquist G. Mental health consultation-liaison interventions in primary care. In: Miranda J, Hohmann A, Attkisson C, Larson D, editors. Mental Disorders in Primary Care. San Francisco, CA: JosseyBass Publishers; 1994. p. 347-73.

30. Lopes G. Serviço Consiliar/Ligação (C/L) de Psiquiatria, Psicossomática e Saúde Mental. Psiquiatria Clínica 1994; 15 (2): 50-64.

\author{
ENDEREÇO PARA CORRESPONDÊNCIA \\ Ana Sofia Cabral \\ Urbanização Varandas Quinta da Lomba, $n^{\circ} 21,2^{\circ}$ Dto \\ 3030-478 Coimbra \\ E-mail: anaprimocabral@hotmail.com
}

Recebido em 20/06/2008

Aceite para publicação em 13/10/2008

\section{ABSTRACT}

It's consensually assumed both the high prevalence of psychiatric pathology in the Primary Care and the fact that the General Practitioner is the best positioned agent to carry out a correct diagnostic and therapeutic approach to these problems. Accordingly, it is suggested that the General Practitioner evaluate and treat the majority of these patients, referring a minority, really needed, to specialized care. In practical terms, it is assumed as a priority that the General Practitioner gradually improves his ability of intervening in the area of Mental Health. Therefore, in this context, the articulation between Psychiatry Services and Primary Care Centers is considered highly rewarding, with the special role of Liaison Psychiatry.

In the present article the authors make some considerations and put different questions on the subject in appraise, trying to approach important issues, mainly related to the specific presentation of psychiatric pathology in Primary Care, particularly the case of «depression», and related difficulties in the evaluation of mental disorders in this setting.

Keywords: Psychiatric Pathology; Primary Care; Consultation/Liaison Psychiatry. 